\title{
Amplitude Dependent Internal Friction and Magneto-Acoustic Effects in YBCO Ceramics
}

\author{
B.K. Kardashev, S.P. Nikanorov, P. Devos*,** and R. De Batist* \\ A.F. Ioffe Physico-Technical Institute of the Russian Academy of Sciences, St. Petersburg, 194021, \\ Russia \\ * University of Antwerp (RUCA), 2020 Antwerpen, Belgium \\ ** Flemish Institute for Technological Research (VITO), $2400 \mathrm{Mol}$, Belgium
}

\begin{abstract}
The paper is concerned with effects in damping and Young's modulus observed in YBaCuO ceramic superconductors vibrating ultrasonically either in or without magnetic field. The amplitude and time dependencies which arise as a result of high amplitude vibrational excitation are presented for coarse-grained and fine-grained specimens. Weak magneto-acoustic effects were observed, and found to be strongly influenced by vibration amplitude and by thermal pre-history of the sample. Analysis of the results shows that, in the vicinity of liquid helium temperature, high amplitude ultrasonic stresses induce structural modifications in these YBaCuO ceramics. Dislocation point defect interaction mechanisms are proposed for explaining the micro-plastic behaviour of the $\mathrm{YBaCuO}$ samples observed in the ultrasonic experiments. The magneto-acoustic effects are discussed in the framework of flux line pinning models.
\end{abstract}

\section{INTRODUCTION}

It was shown earlier $[1,2]$ that the micro-plastic behaviour of YBCO ceramic superconductor materials, as manifested in amplitude dependent damping (ADD) and modulus defect (ADMD), can be ascribed to the vibrational motion of crystal dislocations in the stress field of pinning crystal defects. It was found that the pinning defects can change their state when the specimen is subjected to high amplitude ultrasonic excitation; this change of state is reflected in the observed time dependence of the ADD and ADMD [2]. These time dependencies at a constant level of ultrasonic stress amplitude are a manifestation of the microcreep properties of the material. It is interesting to notice that these brittle YBCO ceramics exhibit microcreep, even at liquid helium temperatures.

Whereas the influence of flux line pinning mechanisms on the acoustic properties of superconductors has been well documented [see, e.g., 3,4], it remains uncertain whether magneto-acoustic effects related to crystal dislocations exist in $\mathrm{HT}_{c}$ superconductors. The pronounced ADD and ADMD effects observed by means of the piezoelectric ultrasonic composite oscillator technique (PUCOT) suggests that this technique may be a suitable way to explore this question. Using longitudinal mechanical vibrations, in the direction of the magnetic flux lines, allows one to reduce the effects of flux line pinning mechanisms $[3,4]$, and thus to obtain the best sensitivity for other magneto-acoustic interaction mechanisms.

In this paper we present results of further low temperature ultrasonic measurements on YBCO ceramics prepared using different precursor powders. Whereas the effect of an external magnetic field up to $~ 1 \mathrm{~T}$ on the amplitude dependent acoustic parameters of these YBCO superconductors is essentially negligible, it was found that in the amplitude independent range weak magneto-acoustic effects can be observed, the nature of which appears to be, however, specimen dependent. This qualitative specimen dependence can be removed as a result of high amplitude ultrasonic excitation, in the amplitude dependent range. Possible models for explaining these observations are briefly discussed.

\section{EXPERIMENTAL}

Superconducting YBCO material was prepared from precursor powders of different origins [1]. Two types of ceramics, labeled $B 1$ and $B 3$ in [1], were used in the present investigation. Two Bl type samples were used: B 1-1, B1-2. Specimen dimensions were approximately $1 * 2.5 * 19 \mathrm{~mm}^{3}$. Average grain sizes were found to be approximately $72 * 22 \mu \mathrm{m}^{2}$ and $47 * 9 \mu \mathrm{m}^{2}$ for BI and B3 type specimens, respectively. 


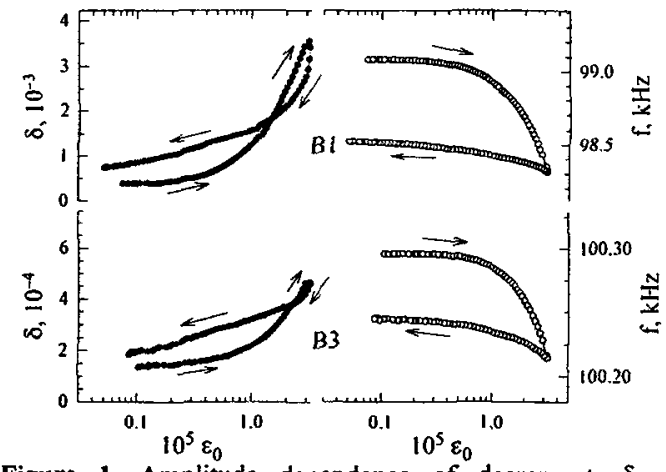

Figure 1. Amplitude dependence of decrement, $\delta$, and resonance frequency, $f$, for coarse grained $(B 1-1)$ and fine grained (B3) samples. The curves were obtained immediately after cooling from room temperature to $6.5 \mathrm{~K}$; arrows indicate the sequence in changing the amplitude. in measurements at a constant low amplitude in the AID range. For the second one, $\delta$ and $\mathrm{f}$ were followed, quasi-simultaneously, at two amplitude levels (in the AI and the AD range), in order to follow their changes under the influence of high amplitude excitation. This approach was possible since it takes no more than 1 to 2 seconds to stabilize the vibration amplitude at any level. Magnetic fields up to $\left|\mu_{0} \mathrm{H}\right|=1.1 \mathrm{~T}$ were applied along the longitudinal vibrational axis of the specimen; the field could be switched on or off at any moment during the experiment.
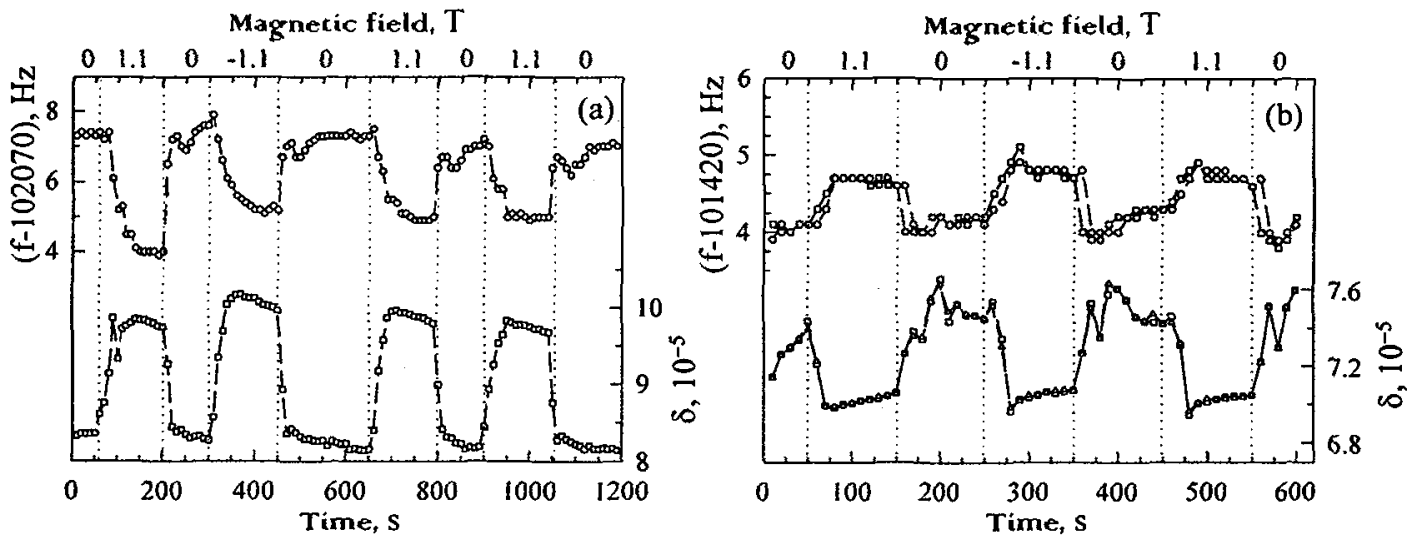

Figure 2. Time diagrams of the experiments at $7 \mathrm{~K}$ with magnetic field of $\pm 1.1 \mathrm{~T}$ for coarse grained $\mathrm{B} /-2$ (a) and fine-grained $\mathrm{B} 3$ (b) samples. Stabilized amplitudes are of the order of $10^{-6}$ in AID range.

\section{RESULTS}

A detailed description of the amplitude dependence of the damping, $\delta(\varepsilon)$, and the resonance frequency, $\mathrm{f}(\varepsilon)$, for three types of YBCO ceramics is given in [1,2]. Here we show, as a reminder, the data for the samples used further in the present investigation, i.e., B1 and B3 (fig.1). Notice the irreversibility occurring between increasing and decreasing vibration amplitudes, testifying to the changes in the microstructure of the specimen induced by high amplitude excitation.

This complicated behaviour of the amplitude dependent damping (ADD) and modulus defect (ADMD, expressed as $(\Delta \mathrm{E} / \mathrm{E})_{\mathrm{h}}$ proportional to $\left.\Delta \mathrm{f} / \mathrm{f}\right)$ makes it rather difficult to investigate possible effects on the acoustic parameters of an additional external (magnetic) field. Nevertheless, as indicated in the "Experimental" section, a procedure could be developed allowing to perform such experiments with high accuracy and sensitivity. 

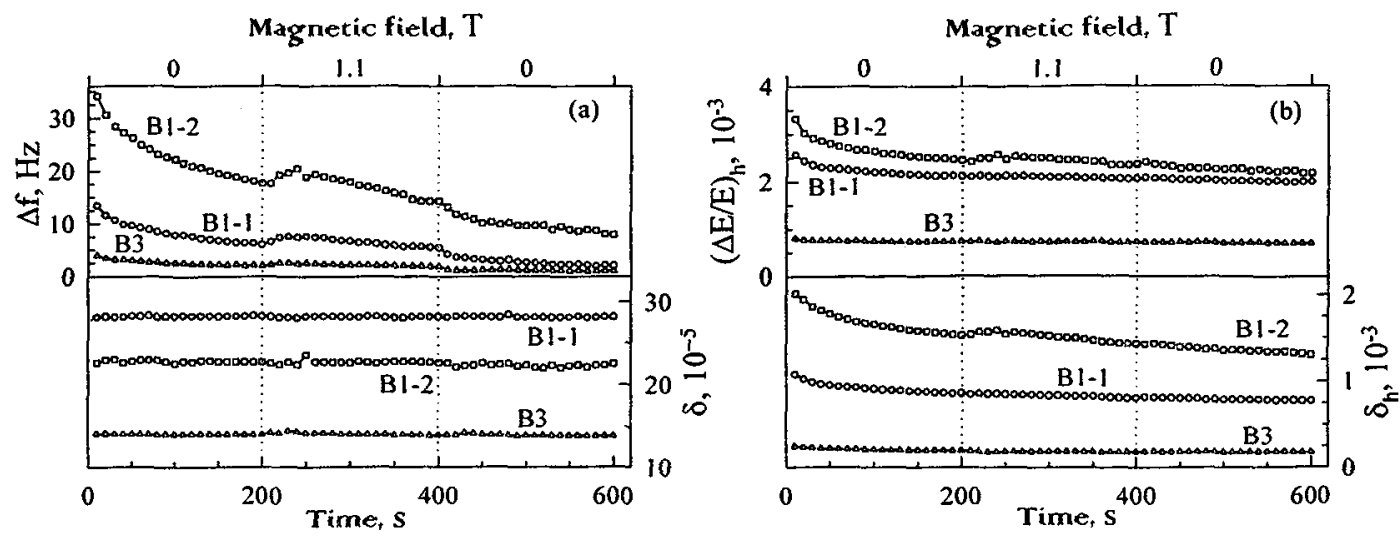

Figure 3. Time dependence of $\delta$ and $f$ in the $A l(a)$ and the $A D(b)$ ranges. These curves were obtained during alternating measurements at low $\left(10^{-6}\right)$ and high $\left(3^{*} 10^{-5}\right)$ amplitudes, using various values of magnetic field.

We describe the experiments and results in chronological order. It should be noted that all samples have already been investigated in low temperature ADD experiments, without magnetic field; they were then stored at room temperature before the present magneto-acoustic experiments were performed. In a first series of measurements, $\delta$ and $f$ were measured at a constant low amplitude, following cooling to $\sim 7 \mathrm{~K}$ in zero magnetic field, to reveal the effect of a magnetic field, applied at $7 \mathrm{~K}$, on the "initial" state of the specimen (i.e. the zero-field cooled state, before high amplitude acoustic excitation). The results for the B1 and B3 type specimens are presented in fig.2. Notice it takes approximately $50 \mathrm{~s}$. for the magnetic field to reach a constant value $( \pm 1.1 \mathrm{~T}, 0 \mathrm{~T})$ after switching the field. Although a change in magnetic field appears to have an undeniable effect on both damping and resonance frequency, the results are found to be distinctly different in nature for the coarse-grained $\mathrm{B} 1$ type material as compared with the fine-grained
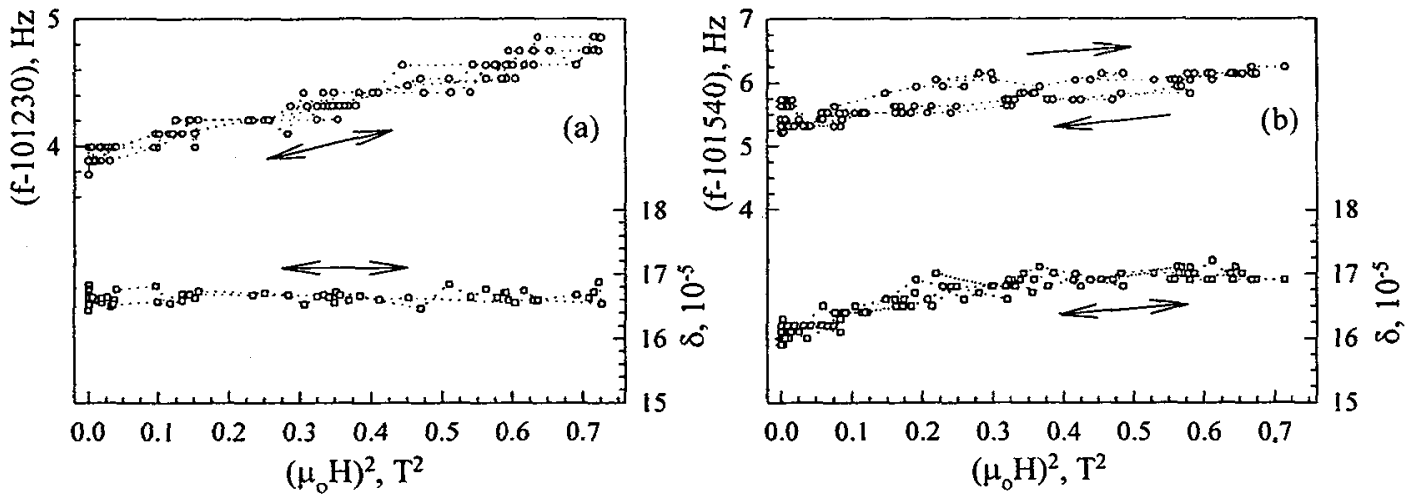

Figure 4. Decrement $\delta$ and resonant frequency $f$ as a function of magnetic fjeld for coarse-grained $B 1-2$ (a) and fine-grained B3 (b) specimens after high amplitude ultrasonic excitation $\left(10 \mathrm{~min} ., \varepsilon=3^{*} 10^{-5}\right) ; \mathrm{T}=8 \mathrm{~K}$.

\section{B3 specimen.}

In the second series of experiments, the effect of high amplitude acoustic excitation was examined by performing acoustic measurements at two levels of strain amplitude $\left(10^{-6} ; 3^{*} 10^{-5}\right)$. As shown in fig.3, the different specimens now show qualitatively the same behaviour, in spite of the quite different initial states revealed in fig.2. The magnetic field dependence of the acoustic parameters in the amplitude independent range, measured following high amplitude excitation (about $10 \mathrm{~min}$. at $3 * 10^{-5}$ ) is shown in fig.4. Finally, it is interesting to notice that the magneto-acoustic properties of specimens B1-1 and B3 could be restored to their initial behaviour by heating to $\sim 100 \mathrm{~K}$, whereas for the $\mathrm{B} 1-2$ specimen the initial state has not been recovered, even after several months at room temperature. 


\section{DISCUSSION}

The main experimental results of this investigation appear to be the following:

1) the effect of magnetic field on the amplitude dependent acoustic parameters is negligible (fig. $3 \mathrm{~b}$ )

2) a longitudinal magnetic field does influence the longitudinal resonance frequency in the amplitude independent range (fig.3a)

3) the magneto-acoustic properties are sensitive to high amplitude acoustic excitation.

The fact that the amplitude dependent acoustic parameters, which are suggested in $[1,2]$ to be a manifestation of dislocation mobility in these YBCO ceramics, are essentially unaffected by magnetic fields is not really unexpected. Indeed, the magnetic field used in these experiments is well below $\mathrm{H}_{\mathrm{c} 2}$, and thus one would not expect the pronounced changes in "normal state" charge carrier concentration required to observe changes in dislocation viscosity [5].

The effect of magnetic field on the acoustic parameters in the amplitude independent range, on the other hand, is difficult to explain in the framework of a dislocation mechanism [5,6]. Indeed, as argued above, the increase in normal state carrier concentration is expected to be quite low and, furthermore, one would not expect the pronounced specimen dependence observed in our experiments. Considering flux line pinning mechanisms $[3,4]$, in general these predict increases in damping and in resonance frequency in the presence of a magnetic field. Whereas the effect on damping is in most of our observations relatively small or even absent, the frequency is found to increase in the presence of a magnetic field in almost all cases (for specimen B1-2, this was so only after high amplitude excitation). On the other hand, for the orientations of the magnetic and mechanical field used in these experiments, no magneto-elastic coupling is expected [3]. The (small) effect observed here could then only be explained by invoking second-order effects related to the ceramic nature of the specimens and the resulting curvature induced in the flux lines. The fact that high amplitude mechanical excitation seems to contribute to stabilizing the magneto-acoustic response can be explained by assuming that dislocation oscillations help the flux lines in approaching the equilibrium pinning state (remember the specimens were zero-field cooled). Yet, the absence of an effect of magnetic field on the amplitude dependent acoustic parameters suggests that dislocations are not really efficient flux line pinners, although the changes in microstructure induced by high amplitude vibrational excitation (in the ADD range) do affect the flux line configuration and result in its stabilization.

Finally, one might also consider magnetostriction effects, possibly related to the flux line pinning mechanisms, to explain the observed magneto-elastic behaviour $[7,8]$, although in this case the effect of vibrational excitation finds no obvious explanation.

\section{CONCLUSION}

Consideration of various magneto-acoustic interaction models indicates that the most probable mechanism for explaining the effects described in this paper is based on the flux line pinning model, in spite of the fact that in first order this magneto-elastic coupling should not be observed for the field orientations used in the present experiments.

\section{Acknowledgments}

This work has been carried out in the frame of the "Incentive Program on high Tc Superconductors" of the Belgian Prime Minister's Services of Scientific, Technical and Cultural Affairs; it was supported in part by a NATO Linkage Grant and by a Research Grant from the Ministry of the Flemish Community. The authors thank Mr. J. Cornelis (VITO) for interesting discussions.

\section{References}

[1] Golyandin S;N., Kardashev B.K., Kustov S.B., Nikanorov S.P., Devos P., Cornelis J., De Batist R., Phys. Stat. Sol. (a), 147 (1995) 111-118.

[2] Kardashev B.K., Golyandin S;N., Kustov S.B., Nikanorov S.P., Devos P.. Cornelis J., De Batist R., Proceedings International Symposium on M3D Ill, ASTM STP 1304 (to be published)

[3] Pankert J., Physica C, 168, (1990) 335-345

[4] Esquinazi P., Journ.LowTemp.Phys., 85 (1991) 139-232.

[5] Schwarz R.B., Isaac R.D. and Granato A.V., Phys.Rev.Lett. 38 (1977) 554-557.

[6] Granato A.V. and Lücke K., J.Appl.Phys., 27 (1956) 583-593.

[7] del Moral A., Ibarra M.R., Algarabel P.A. and Arnaudas J.I., Physica C, 161 (1989) 48-58.

[8] Ikuta H., Hirota N., Nakayama Y., Kishio K. and Kitazawa K., Phys.Rev.Lett., 70 (1993) 2166-2169. 